\title{
Performance Analysis of SAC OCDMA in FSO system using SPD Technique with APD for Different Weather Conditions
}

\author{
Anisha Priyadarshi ${ }^{1}$, Sudipta Mitra ${ }^{2}$ \\ ${ }^{1,2}$ School of Electronics Engineering (SENSE) Vellore Institute of Technology (VIT) Vellore-632014, India
}

\begin{abstract}
Spectral Amplitude Coded-Optical Code Division Multiple Access(SAC-OCDMA) allows several users to transmit simultaneously over the same channel. Each of the users is assigned a code which is a part of orthogonal codes. The channel studied in this research paper is Free Space Optical (FSO) channel FSO has been an area of study in the field of communication because of the varied advantages it offers. FSO channel offers more realistic model in the communication domain. The FSO channel has a limitation that it provides different attenuation for different weather conditions which may include rain, clear, fog and haze weather condition. Hence it is sensitive to the weather condition prevelant in the region. This paper analyses the Bit Error Rate (BER) for different weather conditions. At the receiver side the detection scheme used is Single Photodiode Detection (SPD). Using a single photodiode reduces the shot noise and increases the optical to electrical conversion.
\end{abstract}

Keywords: Spectral Amplitude Coded-Optical Code Division Multiple Access, free space optics, bit error rate, single photodiode detection

\section{Introduction}

Free Space Optical Communication has attracted a number of researches in the field of communication. It allows high bandwidth and effective transmission. It allows point to point communication. SAC-OCDMA system allows several users to transmit signal simultaneously with reliable communication link. OCDMA allows bursty and asynchronous traffic with high flexibility, reliability, scalability and cost effectiveness. OCDMA systems allow several users to transmit simultaneously over a single optical fiber. In OCDMA the information is being transmitted in coded form, so OCDMA systems provide better security against eavesdroppers. In SAC-OCDMA system, as the name suggests, the optical spectrum is amplitude-encoded by a different code for each channel to produce the OCDMA signals. Moreover, these systems can use incoherent light sources such as LEDs. However the FSO channel is affected by weather condition which may include rain, haze, fog etc. leading to a performance degradation. The technique used in the receiver side is Single Photodiode Detection (SPD) technique with Avalanche Photodiode ( APD). Single Photodiode uses detection uses only one photodiode thus reducing the complexity of the receiver and making the system more cost effective. SPD detection technique offers several advantages over other detection technique. It is capable of eliminating both phase induced intensity noise (PIIN) and Multiple Access Interference (MAI).

The given system consistes of three blocks-

\section{System Analysis}

1. Transmitter

2. FSO Channel

3. Receiver

Transmitter- The transmitter side consists of three users. Each user is provided with a bias generator and an LED source. The PN sequence generator is used to generate random signal which is encoded using NRZ encoder. The use of Multiplexer and demultiplexer in the transmitter ensures OCDMA is obtained. The modulator used is Mach Zehnder Modulator. The signal from all the three users is combined by a multiplexer.

FSO Channel- The channel used in the given diagram is FSO. The specification of the FSO can be varied based on different weather conditions.

Receiver- The structure of SAC OCDMA receiver can be seen in figure. The received signal is splitted by using a de-multiplexer. The decoder used is SPD scheme. It consists of two decoders- decoder and an s-decoder. The incoming signal is decoded that has the same spectral response as that of the encoded signal. The decoder detects zero power units for active users and cross corelation power unit for interference. The two interference signals at the optical subtractor are assumed to be equal and ultimately cancel out each other. The output of optical subtractor is either code weight power unit for active user or zero power units for interferer. However, 
practically, there is a slight difference in the two interference signals at the optical subtractor and result in a small amount of optic power which will finally reach the photodiode. SPD detection technique results in the suppression of interference signals in the optical domain. With this, both PIIN and MAI are alleviated in the optical domain. Finally, after the desired signal is detected by a photodiode, the data-carrying electrical signal is low pass filtered by a Bessel filter.

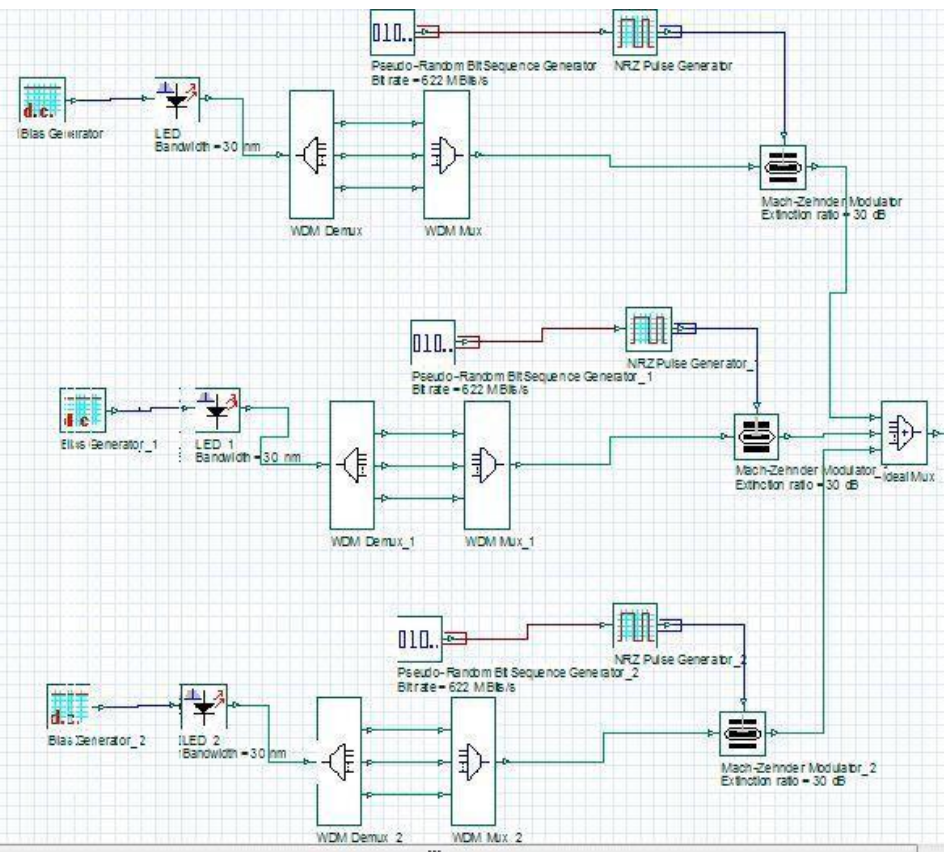

Fig1a- Block Diagram of transmitter

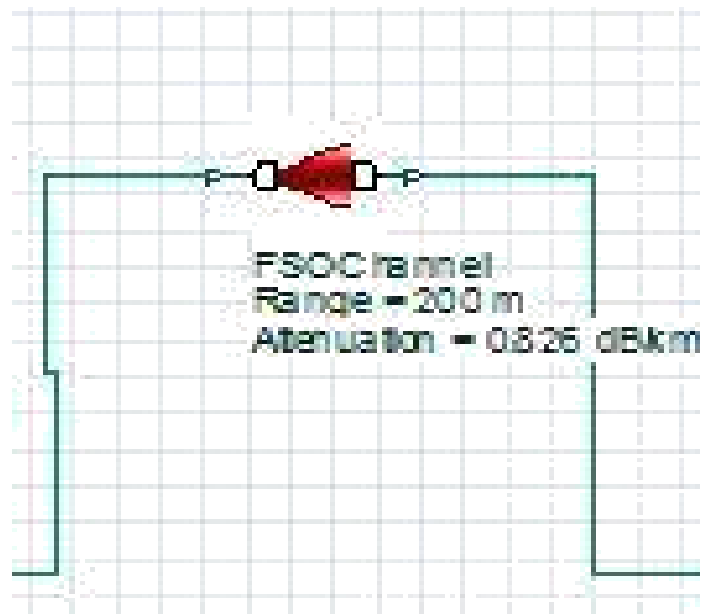

Fig 2b- The FSO channel

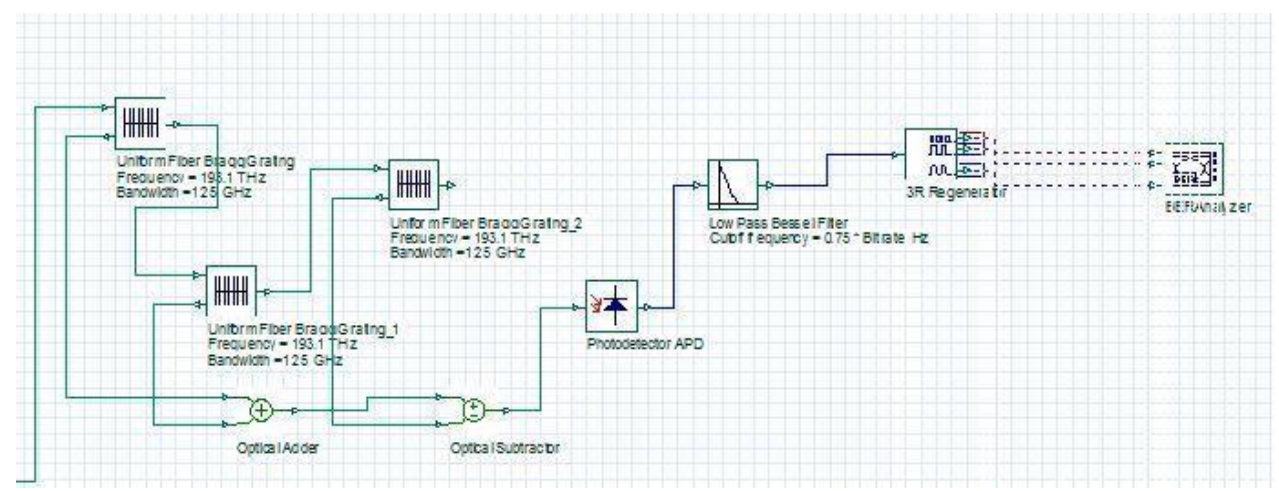

Fig 2c- Reciever of a single user 

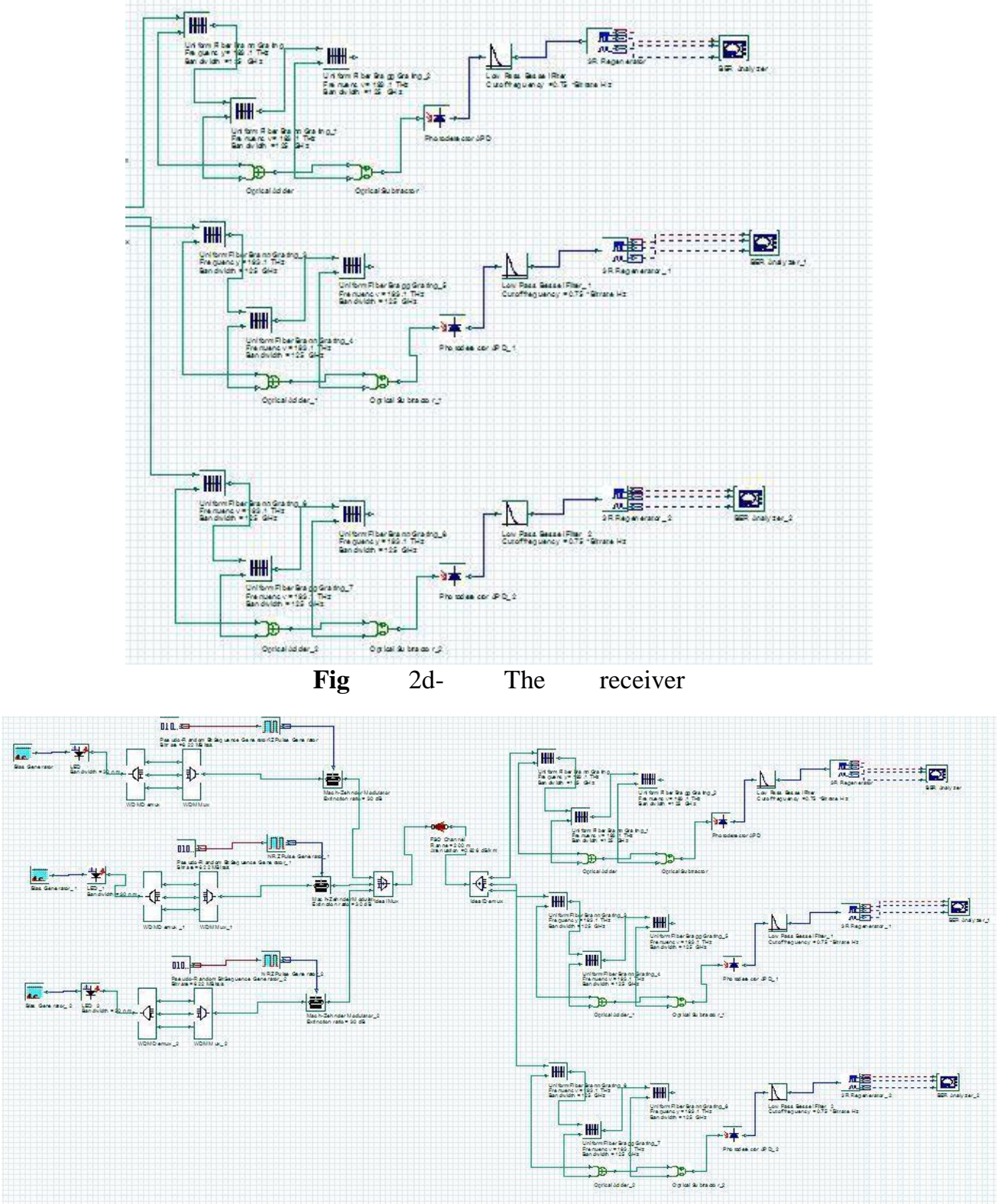

III. Network Simulation And Technical Specifications

The following table shows the technical specifications required for the simulation of the above block diagram.

\begin{tabular}{|l|l|}
\hline LED Bandwidth & $30 \mathrm{~nm}$ \\
\hline LED input power & $9 \mathrm{dBm}$ \\
\hline Bit Rate & $622 \mathrm{Mbps}$ \\
\hline Signal Format & $\mathrm{NRZ}$ \\
\hline External Modulator Extinction & $30 \mathrm{dBm}$ \\
\hline FBGs reflectivity & 0.99 \\
\hline Fiber Dispersion, D & $17 \mathrm{ps} / \mathrm{nm} / \mathrm{km}$ \\
\hline Fiber Dispersion Slope, $\mathrm{S}$ & $0.075 \mathrm{ps} / \mathrm{nm}^{2} / \mathrm{km}$ \\
\hline APD gain & 10 \\
\hline Receiver Filter Bandwidth & $0.75 *$ Bit Rate \\
\hline
\end{tabular}

The above system is simulated for three users for varying channel length of the FSO channel. The length of the channel varies from $200 \mathrm{~m}-1000 \mathrm{~m}$. The system is studies under different kind of weather conditions such as Rain, haze, clear, fog conditions. The different weather conditions affect the channel differently because they have varied attenuation constant. 


\begin{tabular}{|l|l|}
\hline Weather Condition & Attenuation in $\mathrm{dB} / \mathrm{km}$ \\
\hline Rain & 6.27 \\
\hline Haze & 2.37 \\
\hline Clear & 0.233 \\
\hline
\end{tabular}

\section{Results And Discussion}

The Bit error rate (BER) of the system is analyzeed for different weather conditions at a particular length of the FSO channel.
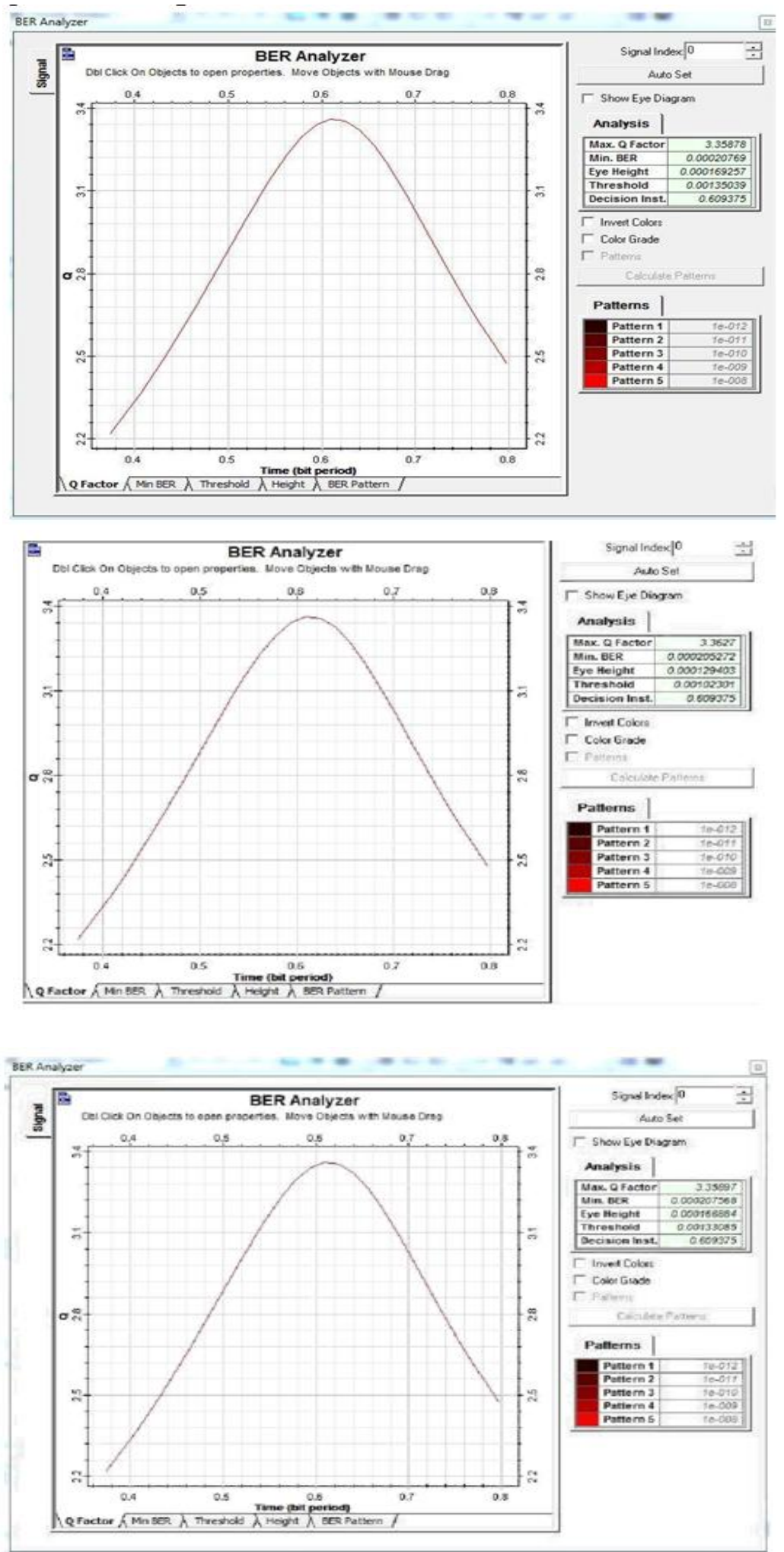
It can be seen and stated clearly from the graph that the maximum Q-factor is obtained in the rain weather condition. Followed by haze and then clear. The minimum BER is of clear weather condition, followed by haze and then rain.

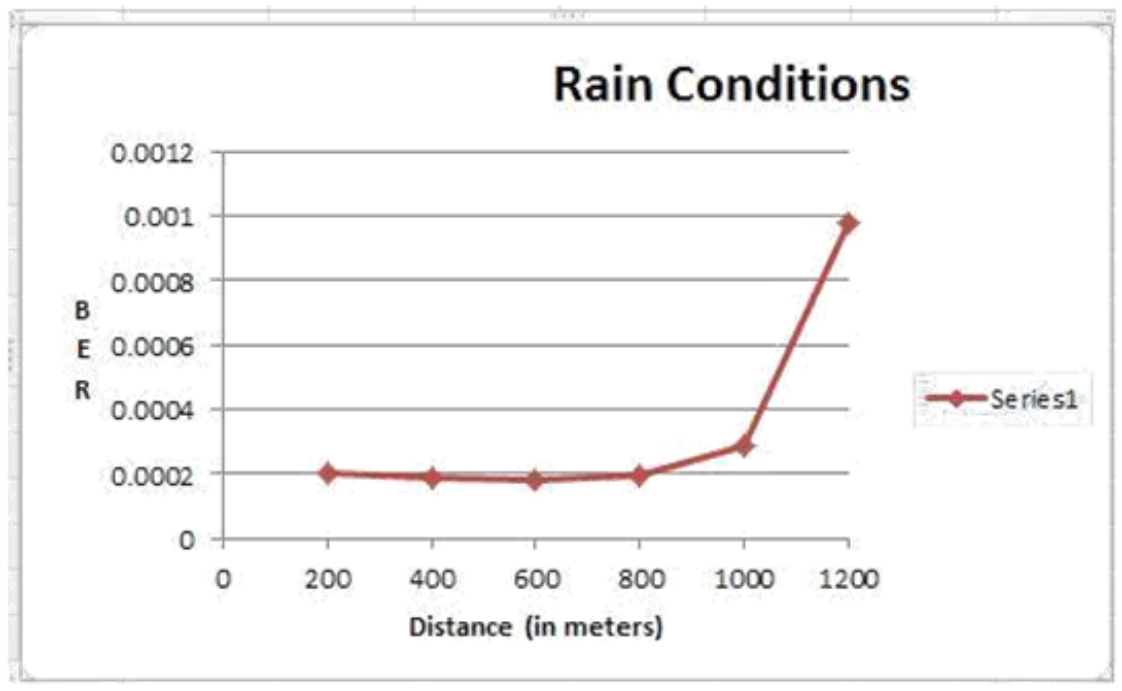

Fig 1 - Distance vs. BER in rain condition

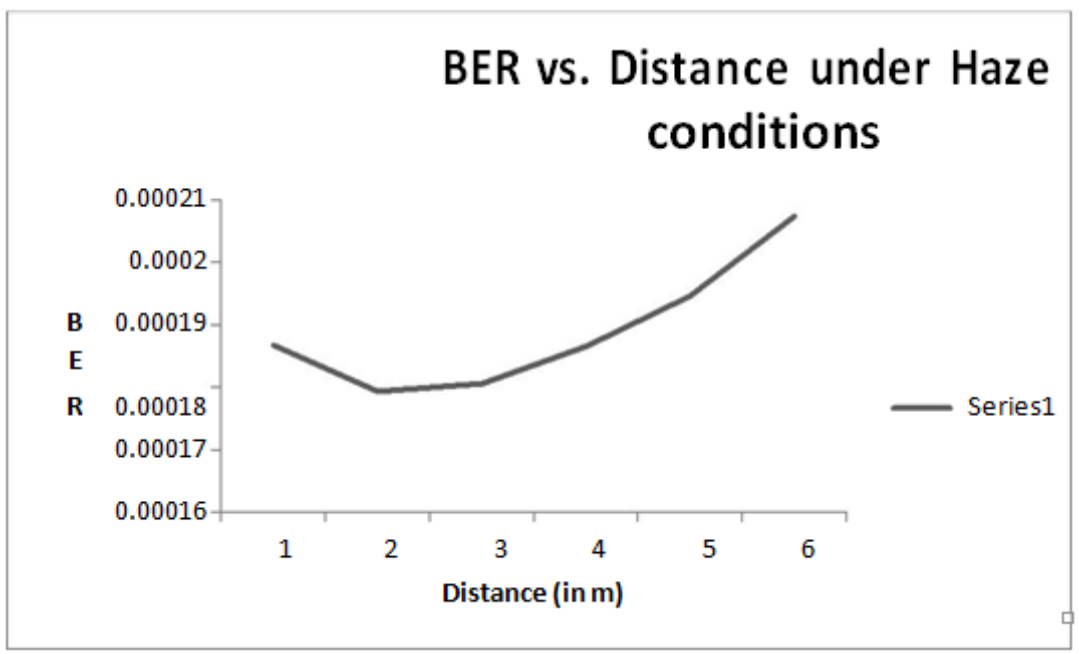

Fig 2- Distance vs. BER in haze condition

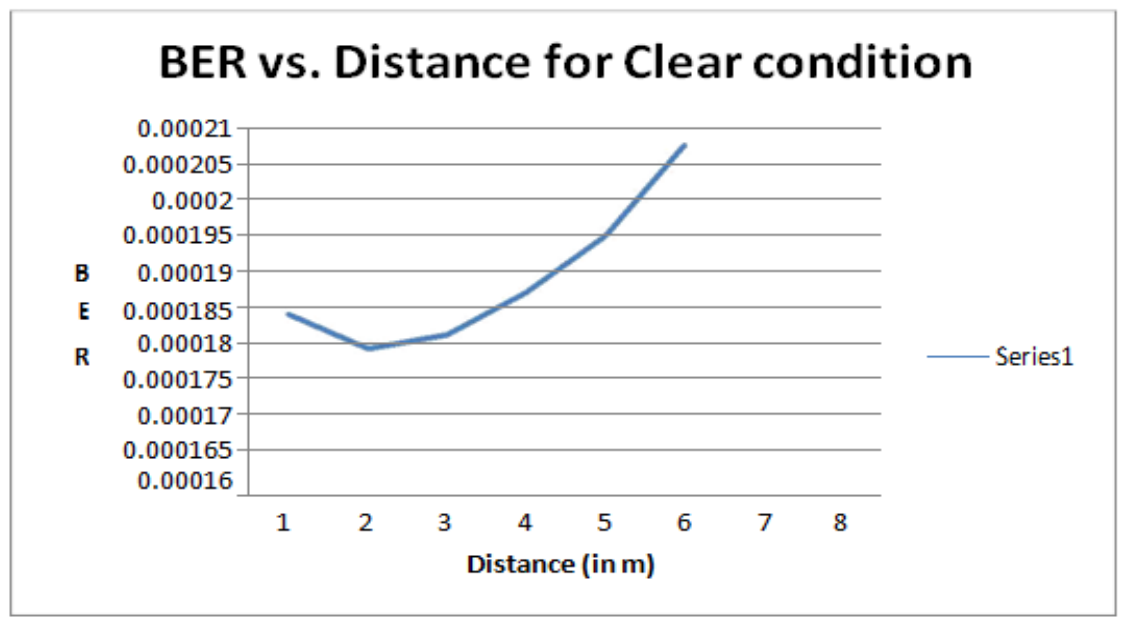

Fig 3- Distance vs. BER in clear weather condition. 
The different weather conditions with different channel length of FSO has been plotted in the figure. It can be seen that the BER of the system increases with increasing the distance between the transmitter and receiver. In fig 1, the condition has been analyzed for rain. In fig 2, condition has been analyzed for haze. In fig 3 , the clear weather analyzed. The BER of the system increases with the increase in haze.

\section{Reverences}

[1] H.S. Mohammed, S.A. Aljunid, H.A. Fadhil, T.H. Abd, Impact of Attenuation on the Hybrid Sub-carrier Multiplexing SAC OCDMA-FSO System, IEEE,2013.

[2] A.G. Alkholidi, K.S. Altowij, Free Space Optical Communications - Theory and Practices, INTECH, 2014.

[3] R.S. Fyath, H.M. Mohammad Ali, Transmission performance of optical code division multiple access network based on spectral amplitude coding,Journal of Emerging Trends in Computing and Information Sciences 3 (3) (2012).

[4] N. Din Keraf, S.A. Aljunid, A.R. Arief, P. Ehkan, The Evolution of Double Weight Codes in Spectral Amplitude Coding OCDMA, Springer, 2015.

[5] H.M.R. Al- Khafaji, S.A. Aljunid, A. Amphawan, H.A. Fadhil, Enhanced probability density function using APD in SAC-OCDMA systems based SPDtechnique, IEEE symposium on industrial electronics and applications (2013).

[6] S. Mostafa, A.N.A. Mohamed, F.E. Abd El- Samie, A.N.Z. Rashed, Comparison Between the Performances of Different Detection Techniques in FreeSpace Optics, IJRECE, 2015.

[7] S.G. Abdulqader, S.A. Aljunid, H.M.R. Al-Khafaji, H.A. Fadhil, Investigation of transmission performance for SAC-OCDMA system under long haultransmission distance based on single photodiode detection technique, IEEE (2013).

[8] Hassan Yousif Ahmed, K.S. Nisar, Diagonal eigenvalue unity code for spectral amplitude coding- optical code division multiple access, Opt. FiberTechnol. (2013)

[9] Z. Jiang, et al., "Four-user, 2.5-Gb/s, spectrally coded OCDMA system demonstration using low-power nonlinear processing," J. LightwaveTechnol., vol. 23, pp. 143-158, 2005.

[10] H.M.R. Al-Khafaji, S.A. Aljunid, A. Amphawan, H.A. Fadhil, and A.M.Safar, "Reducing BER of spectral-amplitude coding optical codedivision multiple-access systems by single photodiode detection technique,' J. Europ. Opt. Soc. Rap. Public., vol. 8, 13022, 2013.

[11] B.S. Naimullah, M. Othman, A.K. Rahman, S.A. Aljunid, Comparison of wavelength propagation for free space optical communications, in: International Conference on Electronic Design, December 1-3, Penang, Malaysia, 2008.

[12] K. Arun, Majumdar, Free-space laser communication performance in the atmospheric channel, J. Optik. Fiber. Commun. Rep. 2 (2005) 345-396, ( 2005 Springer Science. 